PROCEEDINGS OF THE

AMERICAN MATHEMATICAL SOCIETY

Volume 128, Number 6, Pages 1779-1791

S 0002-9939(99)05515-X

Article electronically published on December 8, 1999

\title{
EXISTENCE OF SOLUTIONS FOR FIRST ORDER SINGULAR PROBLEMS
}

\author{
M. CHERPION AND C. DE COSTER
}

(Communicated by Hal L. Smith)

\begin{abstract}
We develop the lower and upper solutions method for first order initial value problems as well as for first order periodic problems in case the nonlinearity presents singularities. More precisely we prove that if we have a lower solution $\alpha$ and an upper solution $\beta$ of these problems, which are not necessarily continuous nor ordered, we have a solution wedged between $\min \{\alpha, \beta\}$ and $\max \{\alpha, \beta\}$.
\end{abstract}

\section{INTRODUCTION}

Let us consider the problem

$$
\left.\left.u^{\prime}=f(t, u) \text { on }\right] 0, T\right], \quad u(0)=0,
$$

where $f:] 0, T] \times] 0,+\infty\left[\rightarrow \mathbb{R}\right.$ is an $L^{1}$-Carathéodory function, singular in $t=0$ and in $u=0$. Due to the singularity, we are not even sure of the local existence of a solution of (1). In this work we develop the lower and upper solutions method for (11).

To our knowledge, the first works devoted to lower and upper solutions for (1) are the papers of G. Peano [20] in 1885 and O. Perron [21] in 1915. They consider the problem (1) with $f$ continuous, assume the existence of continuous functions $\alpha$ and $\beta$ with $\alpha(0)=0=\beta(0), \alpha \leq \beta$ and

$$
D_{ \pm} \alpha(t) \leq f(t, \alpha(t)), \quad D_{ \pm} \beta(t) \geq f(t, \beta(t)),
$$

and prove the existence of a minimal and a maximal solution of (1) between $\alpha$ and $\beta$. The formulation of G. Peano is a little different but the idea is the same. In 1931, G. Scorza Dragoni [23] considers systems and allows $\alpha(0) \leq 0 \leq \beta(0)$. The extension to the Carathéodory case of Scorza Dragoni's result concerning one equation is due to F. Cafiero [3] in 1947. Recently, M. Frigon and D. O'Regan [6] have considered noncontinuous $\alpha$ and $\beta$ and C. Marcelli and P. Rubbioni 15] have studied the situation where $\alpha$ and $\beta$ can cross.

For the periodic problem

$$
u^{\prime}=f(t, u) \text { on }[0, T], \quad u(0)=u(T),
$$

Received by the editors July 24, 1998.

1991 Mathematics Subject Classification. Primary 34A12, 34B15.

Key words and phrases. Singular first order initial value problem, singular first order periodic BVP, lower and upper solutions method. 
we have already found the lower and upper solutions approach in the book of F. Tricomi [24] in 1953, where we also find an application of this approach to a singular problem. In 1958, S. Moretto [18 extends Tricomi's result to a situation where $f$ is a Carathéodory function which satisfies a $L^{1}$-Lipschitz condition and $\alpha$, $\beta$ are supposed to be well ordered $T$-periodic lower and upper solutions. This is extended to systems with $f$ continuous and locally Lipschitz in $u$ by H.W. Knobloch [12] and for $f$ continuous by J. Mawhin [16, 17. The Carathéodory case without Lipschitz condition was considered by A. Adje [1, A. Cabada 2] and M. Nkashama 19]. In [4], C. De Coster considered the Carathéodory case with $\alpha$ and $\beta$ not necessarily continuous.

Except for the book of Tricomi, we have already found the lower and upper solutions method for singular problems in the paper of G. Prodi 22] in 1953 devoted to parabolic equations. More recently, an extensive literature appears on second order O.D.E. with different kind of boundary conditions; see for example C. De Coster, M. Grossinho and P. Habets [5], P. Habets and L. Sanchez 7], P. Habets and F. Zanolin 8, 9, I.T. Kiguradze and B.L. Shekhter [11, T.C. Lee and D. Willett [13], A.G. Lomtatidze [14 and the references therein, but much less is known for problem (11).

In this work we develop the lower and upper solutions method for (1) and for (2), i.e. we prove that if we have a lower solution $\alpha$ and an upper solution $\beta$ of (1) (resp. (21)) which are not necessarily continuous nor ordered, we have a solution of (11) (resp. (2)) between $\min \{\alpha, \beta\}$ and $\max \{\alpha, \beta\}$. The results are illustrated by several examples.

\section{ThE LOWER AND UPPER SOLUTIONS METHOD}

In this section we consider the problem

$$
\left.\left.u^{\prime}=f(t, u) \text { on }\right] 0, T\right], \quad u(0)=0 .
$$

Basic assumptions on $f$ are described in the following definitions.

Definition 2.1. A function $f:] 0, T] \times] 0, \infty[\rightarrow \mathbb{R}$ is a Carathéodory function on $D \subset] 0, T] \times] 0, \infty[$ if

(i) for a.e. $t \in] 0, T]$, the function $f(t,$.$) with domain \{u \in] 0, \infty[:(t, u) \in D\}$ is continuous;

(ii) for all $u \in] 0, \infty[$, the function $f(., u)$ with domain $\{t \in] 0, T]:(t, u) \in D\}$ is measurable.

Definition 2.2. A function $f:] 0, T] \times] 0, \infty\left[\rightarrow \mathbb{R}\right.$ is $L^{1}$-Carathéodory on $D \subset$ ] $0, T] \times] 0, \infty[$ if $f$ is a Carathéodory function on $D$ and

(iii) for all $R>0$, there exists $h_{R} \in L^{1}(0, T)$ such that for a.e. $\left.\left.t \in\right] 0, T\right]$, all $u \in\left[\frac{1}{R}, R\right]$ with $(t, u) \in D$ we have

$$
|f(t, u)| \leq h_{R}(t) .
$$

In case $D=] 0, T] \times] 0, \infty\left[\right.$, we just say that $f$ is $L^{1}$-Carathéodory.

We denote by $W^{1,1}(a, b)$ the set of continuous functions on $[a, b]$ with weak derivative in $L^{1}(a, b)$ and by $W_{l o c}^{1,1}(a, b)$ the set of functions $u \in W^{1,1}(c, d)$ for some $a<c<d<b$.

Let us introduce next the concepts of lower and upper solutions. 
Definition 2.3. A bounded function $\alpha:] 0, T] \rightarrow] 0, \infty[$ is a lower solution of (3) if

(i) $\lim _{t \rightarrow 0} \alpha(t)=0$

(ii) there exist $0=t_{1}<t_{2}<\ldots<t_{n}=T$ such that

(a) for all $i=1, \ldots, n-1, \alpha \in W^{1,1}\left(t_{i}, t_{i+1}\right)$ and for a.e. $\left.t \in\right] t_{i}, t_{i+1}[$,

$$
\alpha^{\prime}(t) \leq f(t, \alpha(t))
$$

(b) for all $i=2, \ldots, n-1$,

$$
\alpha\left(t_{i}\right)=\lim _{t \rightarrow t_{i}^{-}} \alpha(t)>\lim _{t \rightarrow t_{i}^{+}} \alpha(t) .
$$

Definition 2.4. A bounded function $\beta:] 0, T] \rightarrow] 0, \infty[$ is an upper solution of (3) if there exist $0=s_{1}<s_{2}<\ldots<s_{m}=T$ such that

(a) for all $i=1, \ldots, m-1, \beta \in W^{1,1}\left(s_{i}, s_{i+1}\right)$ and for a.e. $\left.t \in\right] s_{i}, s_{i+1}[$,

$$
\beta^{\prime}(t) \geq f(t, \beta(t))
$$

(b) for all $i=2, \ldots, m-1$,

$$
\beta\left(s_{i}\right)=\lim _{t \rightarrow s_{i}^{-}} \beta(t)<\lim _{t \rightarrow s_{i}^{+}} \beta(t) .
$$

Remark 2.1. (i) Observe that, as $\beta(t)>0$ on $] 0, T], \lim _{t \rightarrow 0} \beta(t) \geq 0$.

(ii) In particular, functions $\left.\left.\alpha, \beta \in W^{1,1}(] 0, T\right],\right] 0,+\infty[)$ such that $\alpha(0)=0$ and

$$
\left.\left.\alpha^{\prime}(t) \leq f(t, \alpha(t)), \quad \beta^{\prime}(t) \geq f(t, \beta(t)), \text { a.e. on }\right] 0, T\right],
$$

are respectively lower and upper solutions of (3).

Our main result is the following one.

Theorem 2.1. Let $f:] 0, T] \times] 0, \infty[\rightarrow \mathbb{R}$ and $\alpha$ and $\beta$ be lower and upper solutions of (3). Assume $f$ is $L^{1}$-Carathéodory on

$$
D=\{(t, u) \in] 0, T] \times] 0, \infty[: \min (\alpha(t), \beta(t)) \leq u \leq \max (\alpha(t), \beta(t))\} .
$$

Then the problem (3) has at least one solution $u \in \mathcal{C}([0, T]) \cap W_{\text {loc }}^{1,1}(0, T)$ such that for all $t \in[0, T]$,

$$
\min (\alpha(t), \beta(t)) \leq u(t) \leq \max (\alpha(t), \beta(t)) .
$$

Before proving Theorem 2.1] let us make some comments.

Remark 2.2. Observe that we do not make any assumptions on the number of crossings of $\alpha$ and $\beta$.

Remark 2.3. If $f$ is not $L^{1}$-Carathéodory on $D$, there is no hope to have a solution as shown by the problem

$$
\left.\left.u^{\prime}=-\frac{1}{t}(u-1) \text { on }\right] 0, T\right], \quad u(0)=0 .
$$

This problem has no solution although $\alpha(t)=\frac{t}{2 T}$ and $\beta(t)=2$ are lower and upper solutions of (4).

Remark 2.4. Without additional assumptions, we cannot expect more regularity on the solutions (such as $u \in W^{1,1}(0, T)$ ). We just have to consider the problem

$$
\left.\left.u^{\prime}=f(t, u) \text { on }\right] 0,1\right], \quad u(0)=0,
$$


with

$$
\begin{aligned}
f(t, u) & =2 t\left(2+\sin \frac{1}{t^{2}}\right)-\left(\frac{2\left(2 u-t^{2}\right)}{t^{3}\left(3+2 \sin \frac{1}{t^{2}}\right)}\right) \cos \frac{1}{t^{2}}, & & \text { if } u<t^{2}\left(2+\sin \frac{1}{t^{2}}\right), \\
& =2 t\left(2+\sin \frac{1}{t^{2}}\right)-\left(\frac{2\left(4 t^{2}-u\right)}{t^{3}\left(2-\sin \frac{1}{t^{2}}\right)}\right) \cos \frac{1}{t^{2}}, & & \text { if } u \geq t^{2}\left(2+\sin \frac{1}{t^{2}}\right) .
\end{aligned}
$$

It is easy to observe that $\alpha(t)=\frac{t^{2}}{2}$ is a lower solution, $\beta(t)=4 t^{2}$ is an upper solution and $u(t)=t^{2}\left(2+\sin \frac{1}{t^{2}}\right)$ if $t \neq 0, u(0)=0$ is a solution of (5) with $u \in \mathcal{C}([0,1]) \cap W_{\text {loc }}^{1,1}(0,1)$ but $u \notin W^{1,1}(0,1)$.

Remark 2.5. In case $\alpha$ and $\beta$ cross each other, the situation is quite different according to $f$ being continuous and $\left.\left.\alpha, \beta \in \mathcal{C}^{1}(] 0, T\right]\right)$ or $f$ being just $L^{1}$-Carathéodory and $\alpha, \beta \in W^{1,1}(0, T)$. In the continuous case, if $\alpha$ and $\beta$ cross at $t_{0}$ with $\alpha$ lower than $\beta$ on the left of $t_{0}$ and $\beta$ lower than $\alpha$ on the right of $t_{0}$, we deduce from the definition of lower and upper solutions that $\alpha$ and $\beta$ are tangent at $t_{0}$. This is different in the Carathéodory case as shown by the following example. The problem

$$
\left.\left.u^{\prime}=\frac{(\cos t-2) \sqrt[3]{u-t}}{\sqrt[3]{\sin t}}+2 \text { on }\right] 0, T\right], \quad u(0)=0
$$

has $\alpha(t)=t$ as a lower solution and $\beta(t)=\sin t+t$ as an upper solution. Observe that $\alpha$ and $\beta$ cross at $t=\pi$ as above but without being tangent. Moreover, if $T>2 \pi, \alpha$ and $\beta$ have points where they cross one way and points where they cross the other way.

The next example shows the interest of allowing jumps in the lower and upper solutions.

Example 2.1. Consider the problem

$$
\left.\left.\varepsilon u^{\prime}+u^{3}=f(t) \text { on }\right] 0,2\right], \quad u(0)=0,
$$

where $\varepsilon>0$ is a small parameter and

$$
\begin{aligned}
f(t) & =t^{3}, \quad \text { if } 0<t \leq 1, \\
& =0, \quad \text { if } t>1 .
\end{aligned}
$$

We are interested in the existence of solution $u_{\varepsilon}$ and the asymptotic behaviour of $u_{\varepsilon}$ when $\varepsilon$ tends to zero. It is easy to observe that

$$
\begin{aligned}
\alpha(t) & =\max \left\{0, t-\varepsilon^{1 / 3}\right\}, & & \text { if } 0<t \leq 1, \\
& =0, & & \text { if } t>1,
\end{aligned}
$$

is a lower solution of (6) and

$$
\begin{aligned}
\beta(t) & =t+k, & & \text { if } 0<t \leq 1, \\
& =(1+k)\left(\frac{\varepsilon}{2 t-2+\varepsilon}\right)^{1 / 2}, & & \text { if } t>1,
\end{aligned}
$$

with $k=\frac{\sqrt{\varepsilon}}{2}(\sqrt{2+\varepsilon}+\sqrt{\varepsilon})$ is an upper solution. Hence there is a solution $u_{\varepsilon} \in[\alpha, \beta]$ from which we deduce the pointwise limit

$$
\begin{aligned}
\lim _{\varepsilon \rightarrow 0} u_{\varepsilon}(t) & =t, \quad \text { if } 0<t \leq 1, \\
& =0, \quad \text { if } t>1 .
\end{aligned}
$$

Moreover, as the nonlinearity is decreasing in $u$, it can be proved that the solution $u_{\varepsilon}$ is unique. This is the goal of the next result. 
Theorem 2.2. Let $f:] 0, T] \times] 0, \infty\left[\rightarrow \mathbb{R}\right.$ be $L^{1}$-Carathéodory and assume there exists $L \in L^{1}(0, T)$ such that, for a.e. $\left.\left.t \in\right] 0, T\right]$ and all $\left.u, v \in\right] 0,+\infty[$,

$$
(u-v)(f(t, u)-f(t, v)) \leq L(t)(u-v)^{2} .
$$

Then the problem (3) has at most one solution.

Proof. Let $u$ and $v$ be two solutions of (3). Define $w(t)=\frac{1}{2}(u(t)-v(t))^{2}$. We have that

$$
\begin{aligned}
\frac{d}{d t}(w(t) \exp ( & \left.\left.-2 \int_{0}^{t} L(s) d s\right)\right) \\
& =\exp \left(-2 \int_{0}^{t} L(s) d s\right)\left[(u(t)-v(t))\left(u^{\prime}(t)-v^{\prime}(t)\right)-2 L(t) w(t)\right] \leq 0 .
\end{aligned}
$$

As $u(0)-v(0)=0$, we deduce $u=v$ on $[0, T]$.

Using Theorem 2.1 we can consider repulsive or attractive singular forces.

Example 2.2. The problems

$$
\left.\left.u^{\prime}=\frac{1}{u}-\frac{2}{\sqrt{t}}-\frac{1}{4} \text { on }\right] 0,1\right], \quad u(0)=0,
$$

as well as

$$
\left.\left.u^{\prime}=-\frac{1}{u}+\frac{2}{\sqrt{t}}+\frac{1}{4} \text { on }\right] 0,1\right], \quad u(0)=0
$$

have at least one solution in $W^{1,1}(0,1)$ as $\alpha(t)=\frac{\sqrt{t}}{4}$ and $\beta(t)=4$ are lower and upper solutions of (7) and $\alpha(t)=2 \sqrt{t}$ and $\beta(t)=4 \sqrt{t}$ are lower and upper solutions of (8). We can prove the existence of a second solution for (8) as $\beta(t)=\frac{\sqrt{t}}{4}$ is an upper solution and $\alpha(t)=\sqrt{t}$ is a lower solution. But we deduce from Theorem 2.2 the uniqueness of the solution of problem (7).

Remark 2.6. In the previous example we have a nonlinearity which is singular in $u$, but not really singular in $t$ as for every $u, f(., u) \in L^{1}(0, T)$. We can also consider stronger singularities in $t$ if $\alpha$ and $\beta$ are chosen adequately as shown in the next example.

Example 2.3. Let us consider the problem

$$
\left.\left.u^{\prime}=\frac{1}{u^{2}}-\frac{1}{t^{2}} \text { on }\right] 0,1\right], \quad u(0)=0 .
$$

This problem has a solution, as $\alpha(t)=\frac{t}{2}$ and $\beta(t)=t$ are lower and upper solutions of (9) and $f$ is $L^{1}$-Carathéodory on $\left.\left.D=\{(t, u) \in] 0,1\right] \times\right] 0, \infty\left[: \frac{t}{2} \leq u \leq t\right\}$ even though $f(., u) \notin L^{1}(0,1)$.

Now let us prove Theorem 2.1. To this aim we need the following lemmata.

Lemma 2.3. Let the assumptions of Theorem 2.1 be satisfied and $t_{1}, t_{2}$ be such that $0 \leq t_{1}<t_{2} \leq T$ and $\alpha \leq \beta$ a.e. on $\left[t_{1}, t_{2}\right]$. For every $x_{1}>0$ with $\alpha\left(t_{1}\right) \leq$ $x_{1} \leq \beta\left(t_{1}\right)$, the problem

$$
u^{\prime}=f(t, u), \quad u\left(t_{1}\right)=x_{1},
$$

has at least one solution $u \in W^{1,1}\left(t_{1}, t_{2}\right)$ such that for all $t \in\left[t_{1}, t_{2}\right]$,

$$
\alpha(t) \leq u(t) \leq \beta(t) \text {. }
$$


Proof. Consider the modified problem

$$
u^{\prime}=f(t, \gamma(t, u)), \quad u\left(t_{1}\right)=x_{1},
$$

where $\gamma(t, u)=\alpha(t)+(u-\alpha(t))^{+}-(u-\beta(t))^{+}, u^{+}:=\max \{0, u\}$. Extend $f$ to ] $0,2 T[\times] 0,+\infty[$.

By [10. Theorem I-5.1], there exist $\bar{t}_{1}<t_{1}<\bar{t}_{2} \leq 2 T$ such that the problem (11) has a maximal solution $u:] \bar{t}_{1}, \bar{t}_{2}\left[\rightarrow \mathbb{R}, u \in W_{\text {loc }}^{1, \bar{t}_{1}}, \bar{t}_{2}\right)$. If we prove that on $\left[t_{1}, t_{2}\right] \cap\left[t_{1}, \bar{t}_{2}\left[, \alpha \leq u \leq \beta\right.\right.$, we deduce from [10, Theorem I-5.2] that $\bar{t}_{2}>t_{2}$. So $u \in W^{1,1}\left(t_{1}, t_{2}\right)$ and the result follows as $u$ is also a solution of (10).

Let us prove that $u \geq \alpha$ on $\left[t_{1}, t_{2}\right] \cap\left[t_{1}, \bar{t}_{2}\left[\right.\right.$. If it were not true and as $\alpha\left(t_{1}\right) \leq u\left(t_{1}\right)$ and the jumps of $\alpha$ are downward, then there exist $t_{1} \leq t_{3}<t_{4}<\bar{t}_{2}$ such that $\alpha\left(t_{3}\right)=u\left(t_{3}\right), \alpha \in W^{1,1}\left(t_{3}, t_{4}\right)$ and for all $\left.\left.t \in\right] t_{3}, t_{4}\right], \alpha(t)>u(t)$. This gives us the contradiction

$0>u\left(t_{4}\right)-\alpha\left(t_{4}\right)=\int_{t_{3}}^{t_{4}}\left[u^{\prime}(s)-\alpha^{\prime}(s)\right] d s \geq \int_{t_{3}}^{t_{4}}[f(s, \gamma(s, u(s)))-f(s, \alpha(s))] d s=0$.

In a similar way, we prove that $u \leq \beta$ on $\left[t_{1}, t_{2}\right] \cap\left[t_{1}, \bar{t}_{2}[\right.$.

Lemma 2.4. Let the assumptions of Theorem 2.1 be satisfied and $t_{1}, t_{2}$ be such that $0 \leq t_{1}<t_{2} \leq T$ and $\beta \leq \alpha$ a.e. on $\left[t_{1}, t_{2}\right]$. For every $x_{2}>0$ with $\beta\left(t_{2}\right) \leq$ $x_{2} \leq \alpha\left(t_{2}\right)$, the problem

$$
u^{\prime}=f(t, u), \quad u\left(t_{2}\right)=x_{2},
$$

has at least one solution $u \in W^{1,1}\left(t_{1}, t_{2}\right)$ such that for all $t \in\left[t_{1}, t_{2}\right]$,

$$
\beta(t) \leq u(t) \leq \alpha(t)
$$

Proof. By the change of variables $s=t_{1}+t_{2}-t$, we are reduced to Lemma 2.3

Lemma 2.5. Under the assumptions of Theorem 2.1, for every $t_{1} \in[0, T]$, every $x_{1}>0$ with $\alpha\left(t_{1}\right) \leq x_{1} \leq \beta\left(t_{1}\right)$, the problem

$$
u^{\prime}=f(t, u), \quad u\left(t_{1}\right)=x_{1},
$$

has at least one solution $u \in W^{1,1}\left(t_{1}, T\right)$ such that for all $t \in\left[t_{1}, T\right]$,

$$
\min (\alpha(t), \beta(t)) \leq u(t) \leq \max (\alpha(t), \beta(t)) .
$$

Sketch of the proof. Let $\left.\left.\left.A=\{t \in] t_{1}, T\right] \mid \alpha(t)<\beta(t)\right\}, B=\{t \in] t_{1}, T\right] \mid \beta(t)<$ $\alpha(t)\}$ and $\left.C=] t_{1}, T\right] \backslash(A \cup B)$. It is well known that $\operatorname{int}(A)$ and $\operatorname{int}(B)$ are countable unions of disjoint open intervals, say

$$
\left.\operatorname{int}(A)=\bigcup_{i=1}^{+\infty}\right] a_{i}, b_{i}\left[, \quad \operatorname{int}(B)=\bigcup_{j=1}^{+\infty}\right] c_{j}, d_{j}[.
$$

For $t_{0}=a_{i}$ or $d_{j}$, we consider the nonempty interval

$$
\left[\lim _{t \rightarrow t_{0}^{+}} \alpha(t), \lim _{t \rightarrow t_{0}^{+}} \beta(t)\right] \cap\left[\lim _{t \rightarrow t_{0}^{-}} \beta(t), \lim _{t \rightarrow t_{0}^{-}} \alpha(t)\right]
$$

and we define $m\left(t_{0}\right)$ to be the minimum of this interval if $t_{0} \neq t_{1}$ and $m\left(t_{1}\right)=x_{1}$. On each interval $\left[a_{i}, b_{i}\right]$, we define, from Lemma 2.3 a function $u_{i}$ which is a solution of

$$
u^{\prime}=f(t, u) \text { on }\left[a_{i}, b_{i}\right], \quad u\left(a_{i}\right)=m\left(a_{i}\right)
$$


and such that $\alpha \leq u_{i} \leq \beta$. On each interval $\left[c_{j}, d_{j}\right]$, we define, from Lemma [2.4, a function $v_{j}$ which is a solution of

$$
u^{\prime}=f(t, u) \text { on }\left[c_{j}, d_{j}\right], \quad u\left(d_{j}\right)=m\left(d_{j}\right),
$$

and such that $\beta \leq v_{j} \leq \alpha$.

It can be proved, along the lines of [15], that the function

$$
\begin{aligned}
u(t) & =u_{i}(t), & & \text { if } t \in\left[a_{i}, b_{i}\right] \text { for some } i, \\
& =v_{j}(t), & & \text { if } t \in\left[c_{j}, d_{j}\right] \text { for some } j, \\
& =\alpha(t)=\beta(t), & & \text { otherwise, }
\end{aligned}
$$

is the required solution.

Proof of Theorem 2.1. We consider separately the cases $\lim _{t \rightarrow 0} \beta(t)>0$ or $\lim _{t \rightarrow 0} \beta(t)=$ 0 . Set $A=\{t \in] 0, T] \mid \alpha(t)<\beta(t)\}, B=\{t \in] 0, T] \mid \beta(t)<\alpha(t)\}$ and $C=] 0, T] \backslash(A \cup B)$.

Case 1: $\lim _{t \rightarrow 0} \beta(t)=\bar{\varepsilon}>0$. Let $\left(\varepsilon_{n}\right)_{n}$ be a decreasing sequence of positive numbers such that $\lim _{n \rightarrow \infty} \varepsilon_{n}=0$ and $\varepsilon_{1} \leq \bar{\varepsilon}$. Consider the approximations problem

$$
\left.\left.u^{\prime}=f(t, u) \text { on }\right] 0, T\right], \quad u(0)=\varepsilon_{n} .
$$

As a solution of $\left(P_{n-1}\right)$ is an upper solution of (3), it is not difficult to prove, by induction using Lemma 2.5, that for every $n$, the problem $\left(P_{n}\right)$ has a solution $u_{n}$ such that

$$
\begin{array}{ll}
\min (\alpha, \beta) \leq \ldots \leq u_{n} \leq u_{n-1} \leq \ldots \leq u_{1} \leq \max (\alpha, \beta) & \text { on } A \\
\min (\alpha, \beta) \leq u_{1} \leq \ldots \leq u_{n-1} \leq u_{n} \leq \ldots \leq \max (\alpha, \beta) & \text { on } B
\end{array}
$$

As in 9], we conclude that the pointwise limit of $\left(u_{n}\right)_{n}$ is a function $u \in \mathcal{C}([0, T]) \cap$ $W_{l o c}^{1,1}(0, T)$, a solution of (3) such that, for all $t \in[0, T]$,

$$
\min (\alpha(t), \beta(t)) \leq u(t) \leq \max (\alpha(t), \beta(t)) .
$$

Case 2: $\lim _{t \rightarrow 0} \beta(t)=0$ and there exists a decreasing sequence $\left(t_{n}\right)_{n}$ of positive numbers such that $\lim _{n \rightarrow \infty} t_{n}=0$ and $\beta\left(t_{n}\right) \geq \alpha\left(t_{n}\right)$. Consider the approximations problem

$$
\left.\left.u^{\prime}=f(t, u) \text { on }\right] t_{n}, T\right], \quad u\left(t_{n}\right)=\beta\left(t_{n}\right) .
$$

By Lemma 2.5, the problem $\left(Q_{1}\right)$ has a solution $u_{1} \in W^{1,1}\left(t_{1}, T\right)$ such that for all $t \in\left[t_{1}, T\right], \min (\alpha(t), \beta(t)) \leq u_{1}(t) \leq \max (\alpha(t), \beta(t))$. Let us define $\beta_{1}$, with $\min (\alpha, \beta) \leq \beta_{1} \leq \max (\alpha, \beta)$, an upper solution of (3) in the following way:

$$
\begin{aligned}
\beta_{1}(t) & =\beta(t), & & \text { if } t \in\left[0, t_{1}\right], \\
& =u_{1}(t), & & \text { if } \left.t \in] t_{1}, T\right] .
\end{aligned}
$$

Arguing by induction and using Lemma 2.5, we have a sequence $\left(u_{n}\right)_{n}$ of solutions of $\left(Q_{n}\right)$ and a sequence $\left(\beta_{n}\right)_{n}$ of upper solutions of (3) defined by

$$
\begin{aligned}
\beta_{n}(t) & =\beta(t), & & \text { if } t \in\left[0, t_{n}\right], \\
& =u_{n}(t), & & \text { if } \left.t \in] t_{n}, T\right],
\end{aligned}
$$

such that

$$
\begin{array}{ll}
\min (\alpha, \beta) \leq \ldots \leq \beta_{n} \leq \beta_{n-1} \leq \ldots \leq \beta_{1} \leq \max (\alpha, \beta) & \text { on } A \\
\min (\alpha, \beta) \leq \beta_{1} \leq \ldots \leq \beta_{n-1} \leq \beta_{n} \leq \ldots \leq \max (\alpha, \beta) & \text { on } B
\end{array}
$$

We conclude again by applying the argument of $[9]$. 
Case 3: $\lim _{t \rightarrow 0} \beta(t)=0$ and there exists $\varepsilon>0$ such that for all $\left.\left.t \in\right] 0, \varepsilon\right], \beta(t)<\alpha(t)$. In that case, if $\beta<\alpha$ on ]0,T], by Lemma 2.4 there exists a solution $u$ of

$$
\left.\left.u^{\prime}=f(t, u) \text { on }\right] 0, T\right], \quad u(T)=\beta(T),
$$

with $\beta \leq u \leq \alpha$. As $\beta(0)=\alpha(0)=0, u$ is a solution of (3).

If $\beta$ and $\alpha$ cross, let $\bar{t}=\sup \{t \in[0, T] \mid \beta(s)<\alpha(s)$ for all $s \in[0, t[\}$. By Lemmata 2.4 and 2.5, there exist $u_{1}$ and $u_{2}$ such that

$$
\begin{aligned}
\left.\left.u_{1}^{\prime}=f\left(t, u_{1}\right) \text { on }\right] 0, \bar{t}\right], & u_{1}(\bar{t})=\alpha(\bar{t}), \\
\left.\left.u_{2}^{\prime}=f\left(t, u_{2}\right) \text { on }\right] \bar{t}, T\right], & u_{2}(\bar{t})=\alpha(\bar{t}) .
\end{aligned}
$$

As $\alpha(0)=\beta(0)$, the function

$$
\begin{aligned}
u(t)=u_{1}(t), & \text { if } t \in] 0, \bar{t}], \\
=u_{2}(t), & \text { if } t \in] \bar{t}, T]
\end{aligned}
$$

is the required solution.

\section{Construction of LOWer And UpPer solutions}

We now give conditions to ensure the existence of the required lower and upper solutions.

Proposition 3.1. Let $f:] 0, T] \times] 0, \infty\left[\rightarrow \mathbb{R}\right.$ be $L^{1}$-Carathéodory and assume

$\left(H_{1}\right)$ there exist $a \in W^{1,1}(0, T), L \in L^{1}(0, T)$ such that $a(0) \geq 0, a^{\prime}>0, L>0$ and for a.e. $t \in] 0, T]$, all $u \in] 0, a(t)]$,

$$
f(t, u) \geq L(t) .
$$

Then there exists a lower solution $\alpha$ of (3).

Remark 3.1. Condition $\left(H_{1}\right)$ is equivalent to

$\left(H_{1}^{\prime}\right)$ for every $\left.\left.K \subset\right] 0, T\right]$ compact, there exist $L_{K} \in L^{1}(K), L_{K}>0$ on $K$ and $\varepsilon_{K}>0$ such that for a.e. $t \in K$ and all $\left.\left.u \in\right] 0, \varepsilon_{K}\right]$,

$$
f(t, u) \geq L_{K}(t) \text {. }
$$

Proof. By replacing $L$ by $\min \left\{a^{\prime}, L\right\}$ if necessary we can assume that for all $t \in$ $[0, T], \int_{0}^{t} L(s) d s \leq a(t)$. It is then easy to observe that

$$
\alpha(t)=\int_{0}^{t} L(s) d s
$$

is a lower solution of (3).

Example 3.1. The condition $\left(H_{1}\right)$ is of course satisfied if there exists $\delta>0$ such that $f(t, u) \geq L(t)>0$ on $] 0, T] \times] 0, \delta]$. But it is also satisfied for functions of the type

$$
f(t, u)=\frac{1}{u^{s}}-\frac{1}{t^{r}}
$$

with $r>0$ and $s>0$.

Remark 3.2. We do not really need $L>0$ a.e. on $] 0, T]$ but only that

$$
\left.\left.\int_{0}^{t} L(s) d s>0 \quad \text { on }\right] 0, T\right] .
$$

Now let us give some conditions which ensure the existence of upper solution. 
Proposition 3.2. Let $f:] 0, T] \times] 0, \infty\left[\rightarrow \mathbb{R}\right.$ be $L^{1}$-Carathéodory and assume there exists $R>0$ such that for a.e. $t \in] 0, T]$,

$$
f(t, R) \leq 0 .
$$

Then there exists an upper solution $\beta$ of (3) such that $\beta(t)>0$ on $[0, T]$.

Proof. We choose $\beta(t)=R$.

When $f \geq 0$ on $] 0, T] \times] 0,+\infty[$, we have an upper solution if $f$ does not tend to infinity too quickly when $u$ tends to infinity.

Proposition 3.3. Let $f:] 0, T] \times] 0, \infty\left[\rightarrow \mathbb{R}\right.$ be $L^{1}$-Carathéodory and assume $\left(H_{2}\right)$ there exist $R>0, h \in \mathcal{C}\left(\left[R, \infty[)\right.\right.$ and $q \in L^{1}(0, T)$ such that $h>0, q \geq 0$ and for a.e. $t \in] 0, T]$, all $u \in[R, \infty[$,

$$
f(t, u) \leq q(t) h(u),
$$

and

$$
\int_{0}^{T} q(s) d s<\int_{R}^{\infty} \frac{1}{h(s)} d s .
$$

Then there exists an upper solution $\beta$ of (3) such that $\beta(t)>0$ on $[0, T]$.

Proof. We define $\beta$ to be the solution of

$$
u^{\prime}=q(t) h(u), \quad u(0)=R .
$$

From (15), we deduce that $\beta$ is defined on $[0, T]$. As $\beta \geq R$ on this interval, using (14), we prove it is an upper solution of (3).

Example 3.2. The prototypes for condition $\left(H_{2}\right)$ are nonlinearities $f(t, u)$ of the form

$$
f(t, u)=\frac{u^{s}}{t^{r}}, \quad \text { for } u>>1,
$$

with $0<r<1$ and $s \leq 1$. This restricts the behaviour of $f(t,$.$) for u$ large but not for $u$ near 0 .

\section{Similar RESUlts FOR THE PERIODiC PROBlEM}

Using the ideas of Section 2, we can consider other problems such as the periodic one. In this section, we indicate how to deal with the problem

$$
u^{\prime}=f(t, u), \quad u(0)=u(T) .
$$

Definition 4.1. A bounded function $\alpha:[0, T] \rightarrow \mathbb{R}$ is a lower solution of (16) if condition (ii) of Definition 2.3 is satisfied and

$$
\alpha(0) \leq \alpha(T) .
$$

In the same way, a bounded function $\beta:[0, T] \rightarrow \mathbb{R}$ is an upper solution of (16) if the conditions of Definition 2.4 are satisfied and

$$
\beta(0) \geq \beta(T) .
$$


Theorem 4.1. Let $f: D \subset] 0, T] \times \mathbb{R} \rightarrow \mathbb{R}$ and $\alpha$ and $\beta$ be lower and upper solutions of (16). Assume $E=\{(t, u) \in] 0, T] \times \mathbb{R} \mid \min (\alpha(t), \beta(t)) \leq u \leq$ $\max (\alpha(t), \beta(t))\} \subset D, f$ is Carathéodory on $E$ and there exists $h \in L^{1}(0, T)$ with

$$
|f(t, u)| \leq h(t) \text { on } E \text {. }
$$

Then the problem (16) has at least one solution $u \in \mathcal{C}([0, T]) \cap W^{1,1}(0, T)$ such that for all $t \in[0, T]$,

$$
\min (\alpha(t), \beta(t)) \leq u(t) \leq \max (\alpha(t), \beta(t)) .
$$

Proof. Case 1: $\alpha(0)<\beta(0)$. If $\alpha \leq \beta$ on $[0, T]$, we consider the modified problem

$$
u^{\prime}+u=f(t, \gamma(t, u))+\gamma(t, u), \quad u(0)=u(T),
$$

where $\gamma(t, u)=\alpha(t)+(u-\alpha(t))^{+}-(u-\beta(t))^{+}$. It is easy to prove by Schauder's theorem that (17) has at least one solution. The proof that the solution $u$ of (17) satisfies $\alpha \leq u \leq \beta$ on $[0, T]$ uses the same ideas as Lemma 2.3

If $\beta(T) \leq \alpha(T)$, let $\bar{t}=\sup \{t \in[0, T] \mid \alpha(s)<\beta(s)$ for all $s \in[0, t[\}$. Observe that, by the boundary restrictions imposed on $\alpha$ and $\beta$, there exists $\bar{x} \in$ $[\alpha(0), \beta(0)] \cap[\beta(T), \alpha(T)]$. By Lemma 2.3 , there exists a solution $u_{1}$ of

$$
\left.\left.u^{\prime}=f(t, u) \text { on }\right] 0, \bar{t}\right], \quad u(0)=\bar{x},
$$

and, by the change of variables $s=\bar{t}+T-t$, we deduce from Lemma2.5 that there exists a solution $u_{2}$ of

$$
\left.\left.u^{\prime}=f(t, u) \text { on }\right] \bar{t}, T\right], \quad u(T)=\bar{x} .
$$

Hence, the result is proved with

$$
\begin{aligned}
u(t)=u_{1}(t), \quad \text { if } t \in[0, \bar{t}], \\
\left.\left.=u_{2}(t), \quad \text { if } t \in\right] \bar{t}, T\right] .
\end{aligned}
$$

If $\beta(T)>\alpha(T)$ and $\alpha \not \leq \beta$, let $\bar{t}$ be defined as above. We have $[\alpha(T), \beta(T)] \subset$ $[\alpha(0), \beta(0)]$ and using Lemmata 2.5 and 2.3, we prove the existence of functions $u_{2}$ and $u_{1}$ such that

$$
\left.\left.u_{2}^{\prime}=f\left(t, u_{2}\right) \text { on }\right] \bar{t}, T\right], \quad u_{2}(\bar{t})=\alpha(\bar{t})=\beta(\bar{t}),
$$

and

$$
\left.\left.u_{1}^{\prime}=f\left(t, u_{1}\right) \text { on }\right] 0, \bar{t}\right], \quad u_{1}(0)=u_{2}(T) .
$$

We conclude as in the previous situation.

Case 2: $\alpha(0)=\beta(0)$. As $\beta(T) \leq \beta(0)=\alpha(0) \leq \alpha(T)$, the solution $u$ of

$$
\left.\left.u^{\prime}=f(t, u) \text { on }\right] 0, T\right], \quad u(T)=\alpha(0)=\beta(0),
$$

given by Lemma 2.5 is periodic.

Case 3: $\alpha(0)>\beta(0)$. The boundary conditions imply $\alpha(T)>\beta(T)$ and we are reduced to Case 1 by the change of variables $s=T-t$.

Example 4.1. Consider the equivalent of problems (17) and (8), i.e.

$$
u^{\prime}=\frac{1}{u}-\frac{2}{\sqrt{t}}-\frac{1}{4}, \quad u(0)=u(1)
$$

as well as

$$
u^{\prime}=-\frac{1}{u}+\frac{2}{\sqrt{t}}+\frac{1}{4}, \quad u(0)=u(1)
$$


We can prove that both (18) and (19) have at most one solution. Such a proof relies on an argument which recalls the proof of Theorem [2.2. Moreover $\alpha(t)=\frac{\sqrt{t}}{4}$ and $\beta(t)=4$ are lower and upper solutions of (18), $\alpha(t)=4$ and $\beta(t)=\frac{\sqrt{t}}{4}$ if $t \leq \frac{1}{2}$, $\beta(t)=\frac{\sqrt{1-t}}{4}$ if $t>\frac{1}{2}$ are lower and upper solutions of (19) and we apply Theorem 4.1 to prove that (18) and (19) have a unique solution. Observe that this situation is quite different from the one observed for the Cauchy problem.

Remark 4.1. Observe that in Theorem 4.1 we allow less singularities than in Theorem 2.1. This is due to the fact that, in applications, lower and upper solutions for the periodic case can usually be chosen "far enough" from the singularity as in the next result. Nevertheless, more singularity as in Theorem 2.1] can be considered in the periodic case also in the following way. First observe that in Theorem 2.1 we allow more singularity mainly in case $\alpha(0)=\beta(0)=0$. In the periodic case, this implies $\beta(T)=0$. Hence two cases have to be considered. Either $\alpha(T)=0$ and the result follows by arguments similar to Theorem 2.1 as the solution of (3) with $\min (\alpha, \beta) \leq u \leq \max (\alpha, \beta)$ has to satisfy $u(T)=0=u(0)$, or in the case $\alpha(T)>0$, we define $\bar{t}=\inf \{t \mid \beta(s)<\alpha(s)$ for all $s \in] t, T]\}$ and again arguing as in Section 2 , we prove the existence of a solution $u_{1}$ of

$$
\left.\left.u^{\prime}=f(t, u) \text { on }\right] 0, \bar{t}\right], \quad u(0)=0,
$$

with $\min (\alpha, \beta) \leq u_{1} \leq \max (\alpha, \beta)$ and of a solution $u_{2}$ of

$$
\left.u^{\prime}=f(t, u) \text { on }\right] \bar{t}, T[, \quad u(T)=0,
$$

with $\beta \leq u_{2} \leq \alpha$ on $[\bar{t}, T]$. Hence,

$$
\begin{aligned}
u(t)=u_{1}(t), & \text { if } t \in[0, \bar{t}], \\
& \left.\left.=u_{2}(t), \quad \text { if } t \in\right] \bar{t}, T\right],
\end{aligned}
$$

is the required solution.

To conclude, consider the following result for a singular periodic problem.

Theorem 4.2. Let $f:[0, T] \times] 0, \infty\left[\rightarrow \mathbb{R}\right.$ be $L^{1}$-Carathéodory. Assume

(i) for some $\varepsilon>0$, $\liminf _{u \rightarrow 0^{+}} f(t, u)>\varepsilon$ uniformly in $t \in[0, T]$;

(ii) there exist $R>0$ and $g_{0} \in L^{1}(0, T)$ such that for a.e. $t \in[0, T]$, all $u \geq R$,

$$
f(t, u) \leq g_{0}(t),
$$

and

$$
\int_{0}^{T} g_{0}(t) d t \leq 0
$$

Then the problem (16) has at least one solution.

Proof. Notice that if $\alpha<R$ is a sufficiently small positive constant, it is a lower solution of (16).

Let $w$ be the solution of

$$
u^{\prime}=g_{0}(t)-\frac{1}{T} \int_{0}^{T} g_{0}(t) d t, \quad u(0)=u(T) .
$$

It is easy to see that for $K>0$ large enough, $\beta(t)=w(t)+K$ is an upper solution of (16) such that $\beta>R>\alpha$ on $[0, T]$. We conclude by Theorem 4.1. 
Using lower and upper solutions with the reversed order according to Theorem 4.2, we can also consider the dual result.

Example 4.2. Model examples for Theorems 4.2 and its dual version are the problems

$$
u^{\prime}=\frac{1}{u}-g(t), \quad u(0)=u(T),
$$

and

$$
u^{\prime}=-\frac{1}{u}+g(t), \quad u(0)=u(T)
$$

where $g \in L^{\infty}(0, T)$ with $\int_{0}^{T} g(t) d t>0$.

\section{REFERENCES}

[1] A. Adje, Existence et multiplicité des solutions d'équations différentielles ordinaires du premier ordre à non-linéarité discontinue, Annales de la Société Scientifique de Bruxelles 101 (1987), 69-87. MR 90h:34017

[2] A. Cabada, The monotone method for first order problems with linear and nonlinear boundary conditions, Appl. Math. and Comp. 63 (1994), 163-186. MR 95h:34032

[3] F. Cafiero, Su un problema ai limiti relativo all'equazione $y^{\prime}=f(x, y, \lambda)$, Giornale di Mat. di Battaglini 77 (1947), 145-163. MR 10:194f

[4] C. De Coster, La méthode des sur et sous solutions dans l'étude de problèmes aux limites, Thèse de doctorat, Louvain-la-Neuve, 1994.

[5] C. De Coster, M. R. Grossinho and P. Habets, On pairs of positive solutions for a singular boundary value problem, Applicable Analysis 59 (1995), 241-256. [MR 97d:34016

[6] M. Frigon and D. O'Regan, Existence results for some initial and boundary value problems without growth restriction, Proc. A.M.S. 123 (1995), 207-216. MR 95c:34035

[7] P. Habets and L. Sanchez, Periodic solutions of some Liénard equations with singularities, Proc. A.M.S. 109 (1990), 1035-1044. MR 90k:34049

[8] P. Habets and F. Zanolin, Upper and lower solutions for a generalized Emden-Fowler equation, J. Math. Anal. Appl. 181 (1994), 684-700. MR 95g:34034

[9] P. Habets and F. Zanolin, Positive solutions for a class of singular boundary value problem, Boll. U.M.I. 9A (1995), 273-286. MR 96j:34031

[10] J.K. Hale, Ordinary differential equations, Wiley-Intersciences, New-York (1969).

[11] I.T. Kiguradze and B.L. Shekhter, Singular boundary-value problems for ordinary second-order differential equations, Itogi Nauki i Tekhniki, Seriya Sovremennye Problemy Matematiki, Noveishie Dostizheniya 30 (1987), 105-201, translated in J. Soviet Math. 43 (1988), 2340-2417. MR 89f:34022

[12] H.W. Knobloch, An existence theorem for periodic solutions on nonlinear ordinary differential equations, Michigan Math. J. 9 (1962), 303-309. MR 26:2694

[13] T.C. Lee and D. Willett, Second order singular boundary value problems, SIAM J. Math. Anal. 8 (1977), 741-755. MR 55:12988

[14] A.G. Lomtatidze, Positive solutions of boundary value problems for second order ODE with singular points, Diff. Urav. 23 (1987), 1685-1692. MR 89d:34040

[15] C. Marcelli and P. Rubbioni, A new extension of classical Müller's theorem, Nonlinear Anal. T.M.A. 28 (1997), 1759-1767. MR 97k:34003

[16] J. Mawhin, Nonlinear functional analysis and periodic solutions of ordinary differential equations, Summer School "Difford 74", Stara Lesna, Czechoslovaquia (1974), 37-60.

[17] J. Mawhin, Nonlinear perturbations of Fredholm mappings in normed spaces and applications to differential equations, Univ. de Brasilia, Trabalho de Matem. 61 (1974).

[18] S. Moretto, Sull'esistenza di soluzioni periodiche per l'equazione $y^{\prime}=f(x, y)$, Annali Univ. Ferrara 8 (1958-1959), 61-67. MR 22:6914

[19] M.N. Nkashama, A generalized upper and lower solutions method and multiplicity results for nonlinear first-order ordinary differential equations, J. Math. Anal. Appl. 140 (1989), 381-395. MR 90e:34006 
[20] G. Peano, Sull'integrabilità delle equazioni differenziali di primo ordine, Atti Acad. Torino 21 (1885), 677-685.

[21] O. Perron, Ein neuer existenzbeweis für die integrale der differentialgleichung $y^{\prime}=f(x, y)$, Math. Ann. 76 (1915), 471-484.

[22] G. Prodi, Teoremi di esistenza per equazioni alle derivate parziali non lineari di tipo parabolico I, II, Rend. Ist. Lombardo 86 (1953), 1-47. MR 16:259c

[23] G. Scorza Dragoni, Sugli integrali dei sistemi di equazioni differenziali, Rend. Ist. Lombardo de Sc. e Let. 64 (1931), 659-682.

[24] F. Tricomi, Equazioni differenziali 2e Ed., Torino (1953). MR 15:793a

Université Catholique de Louvain, Institut de Mathématique Pure et Appliquée, Chemin du Cyclotron 2, 1348 Louvain-La-Neuve, Belgique

E-mail address: cherpion@amm.ucl.ac.be

Université du Littoral - Côte d'Opale, Centre Universitaire de la Mi-Voix, 50 Rue

F. Buisson, B.P. 699, 62228 Calais Cédex, France

E-mail address: decoster@lma.univ-littoral.fr 